\title{
Control of strong-field ionization in ferroelectric lithium niobate: Role of the spontaneous polarization
}

\author{
Vincent Wanie $\odot,{ }^{1,2,3}$ Tian-Jiao Shao, ${ }^{4,5}$ Philippe Lassonde, ${ }^{1}$ Heide Ibrahim $\odot,{ }^{1}$ Jude Deschamps $\odot,{ }^{1}$ Jia-Qi Liu ${ }^{4,5}$ \\ Fabian Ambriz Vargas, ${ }^{1}$ François Vidal $\odot,{ }^{1}$ Andreas Ruediger, ${ }^{1}$ Francesca Calegari, ${ }^{2,3,6}$ Xue-Bin Bian $\odot,{ }^{5, *}$ \\ and François Légaré ${ }^{1, \dagger}$ \\ ${ }^{1}$ Centre Énergie Matériaux Télécommunications, Institut National de la Recherche Scientifique, Varennes J3X1S2, Canada \\ ${ }^{2}$ Center for Free-Electron Laser Science, DESY, Hamburg 22607, Germany \\ ${ }^{3}$ Institute for Photonics and Nanotechnologies CNR-IFN, Milano 20133, Italy \\ ${ }^{4}$ University of Chinese Academy of Sciences, Beijing 100049, China \\ ${ }^{5}$ State Key Laboratory of Magnetic Resonance and Atomic and Molecular Physics, Wuhan Institute of Physics and Mathematics, \\ Innovation Academy for Precision Measurement Science and Technology, Chinese Academy of Sciences, Wuhan 430071, China \\ ${ }^{6}$ Physics Department, University of Hamburg, Hamburg 22761, Germany
}

(Received 16 August 2019; revised manuscript received 10 March 2020; accepted 20 March 2020;

published 7 May 2020)

\begin{abstract}
We report the control of tunnel ionization in lithium niobate $\left(\mathrm{LiNbO}_{3}\right)$ using phase-controlled two-color laser fields. Through a macroscopic observable of high contrast, we disclose the crucial contribution of the microscopic spontaneous polarization of the ferroelectric material to the ionization rate: as the relative two-color phase is varied, the ablated area of $\mathrm{LiNbO}_{3}$ is modulated by $35 \%$ when the laser and crystal polarization directions are parallel. Rotating the sample by $180^{\circ}$ around the laser propagation axis leads to an out-of-phase modulation. We use a two-band model to highlight the key contribution of the material's spontaneous polarization for the symmetry breaking of the ionization rate. Our results open new perspectives for the direct control of ionization dynamics in solids by tailoring the electric field of femtosecond laser pulses.
\end{abstract}

DOI: $10.1103 /$ PhysRevB.101.184103

\section{INTRODUCTION}

Strong-field ionization (SFI) of atoms and molecules with intense laser pulses has been widely investigated in the last decades [1-3] and led to an in-depth understanding of several photoinduced ultrafast processes [4]. One of the most striking outcomes is the emergence of attosecond ( 1 as = $10^{-18} \mathrm{~s}$ ) science, which now allows tracking electrons on their natural timescale [5]. In semiconductor and dielectric materials, SFI is the primary step for phenomena such as refractive index changes [6], high-harmonic generation [7,8], and in the extreme case, optical breakdown [9] triggering laser-induced ablation (LIA) [10,11]. For both, atoms and solids, Keldysh developed a common theory 55 years ago in which an adiabatic parameter $\gamma$ is delimiting the perturbative regime $(\gamma>1)$ from the tunneling one $(\gamma<1)$ [3]. While the gas phase is well elucidated, experiments in solids focused mainly on interactions within the perturbative limit. Only a very few studies have explored the tunneling regime [12-16], even though this number is expected to increase rapidly due to the recent availability of low-frequency laser pulses in the infrared (IR) spectral range [17]. Efforts in that direction are of significant importance since various materials with large band gap compared to IR photon energies are promising candidates

\footnotetext{
*xuebin.bian@wipm.ac.cn

$\dagger$ †egare@emt.inrs.ca
}

for numerous technological applications. For instance, lithium niobate $\left(\mathrm{LiNbO}_{3}\right)$ and chalcogenide glasses are notable compounds used in optical switches and IR detectors $[18,19]$.

Many questions remain to be answered to link the wellunderstood gas phase to the solid state. Of particular interest is the case of noncentrosymmetric systems due to their permanent polarization. It is known that the ionization rate of noncentrosymmetric diatomics in the tunneling regime critically depends on the orientation of the molecular axis relative to the polarization of the laser field [20]. Moreover, strong-field ionization of asymmetric gas molecules typically displays preferential emission directions in molecular frame photoelectron angular distributions (MF-PADs), depending on whether the permanent dipole moment is parallel or antiparallel to the laser field [21]. For crystals, the atomic composition and configuration of the unit cell define their band structure and physical properties. The question naturally arises whether there are similar responses to SFI for noncentrosymmetric gas molecules and materials. Furthermore, can waveform shaping of the laser electric field lead to control over SFI as in the molecular case [22-25]? In this article, we extend the understanding of SFI to the solid state by investigating the interaction between intense femtosecond laser pulses and $\mathrm{LiNbO}_{3}$, a synthetic material employed in several devices due to its ferroelectricity and distinctive optical properties [26]. We use the sensitivity of LIA to probe and control SFI in the tunneling regime by measuring the ablated area at the surface of the material as a function of the relative 
(a)

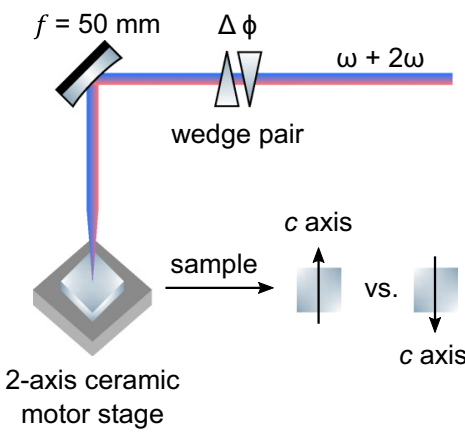

(b)
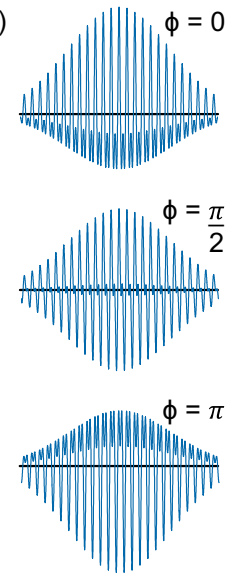

FIG. 1. (a) Sketch of the experimental setup. A laser field composed of the superposition of a fundamental frequency $\omega(\lambda=1800$ $\mathrm{nm})$ and its second harmonic $2 \omega(\lambda=900 \mathrm{~nm})$ is employed to ablate the surface of $\mathrm{LiNbO}_{3}$ samples just above the threshold fluence. The relative two-color phase $\phi$ is adjusted by translating a motorized wedge in a direction perpendicular to the laser propagation axis and the beam is focused onto the crystal with an off-axis parabolic mirror. A fast ceramic motor stage ensures single-shot ablation by translating the sample in a direction parallel to its surface. The laser polarization and the $c$ axis of the crystal are oriented vertically. After a first ablation series, the sample is rotated by $180^{\circ}$ and the procedure is repeated. (b) Waveform representation of the electric field for $\phi=0, \frac{\pi}{2}, \pi$ [Eq. (1)]. The direction of the peak field with respect to the $c$ axis of $\mathrm{LiNbO}_{3}$ changes every $\Delta \phi=\pi$.

phase between a fundamental field $(1800 \mathrm{~nm})$ and its second harmonic $(900 \mathrm{~nm})$. The experimental results are supported by numerical simulations based on a two-band model, which establish the role of the spontaneous polarization of $\mathrm{LiNbO}_{3}$ during the photoionization process.

\section{EXPERIMENTS}

For the experiments presented in Fig. 1(a), the $2.5 \mathrm{kHz}$ (30 fs) Ti:sapphire laser system of the Advanced Laser Light Source (Canada) served as the input for pumping a whitelight seeded optical parametric amplifier (TOPAS, Light Conversion) which delivered the 1800-nm pulses. After spatial filtering, $350 \mathrm{~mW}(140 \mu \mathrm{J})$ were used for secondharmonic generation (SHG) in a type- $1 \beta-\mathrm{BaB}_{2} \mathrm{O}_{4}(\mathrm{BBO})$ crystal $\left(\Theta=20^{\circ}, \Phi=0^{\circ}\right)$ and both beams were sent to a Mach-Zehnder-like interferometer. Spectral separation of the 1800- and 900-nm beams was achieved by a low-dispersion dichroic beam splitter (LaserOptik). The laser polarization angle and energy were controlled in each interferometer arm using a combination of polarizer and half-waveplate. The fundamental and SH fields were recombined through a second dichroic beam splitter and sent through a pair of wedges to adjust and scan their relative phase $\phi$, which was actively stabilized in a similar scheme as in Refs. [27,28]. To avoid chromatic aberrations, the resulting two-color pulses were focused at the surface of $10 \times 10 \mathrm{~mm} \mathrm{Y-cut} \mathrm{LiNbO}_{3}$ samples (MTI Corporation, Curie temperature $T_{c} \simeq 1160^{\circ} \mathrm{C}$ ) by a 50-mm focal length off-axis parabolic gold mirror. The surface thickness was $0.5 \mathrm{~mm}$ and its roughness below $8 \AA$. The

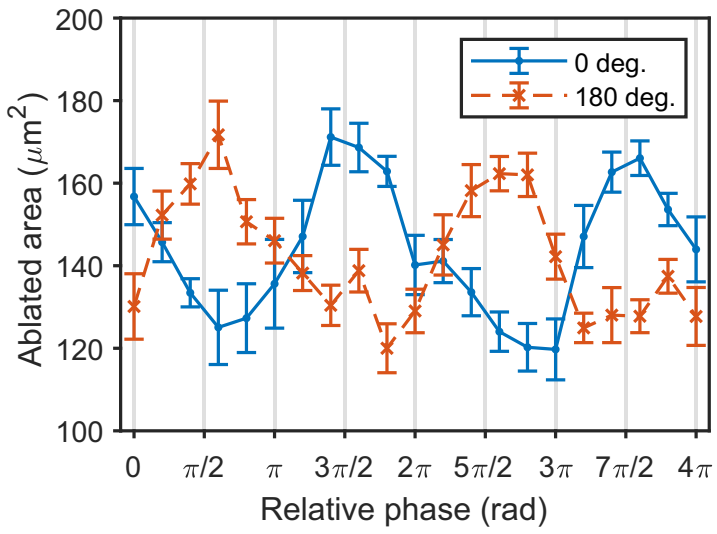

FIG. 2. Single-shot ablated area as a function of the relative twocolor phase for $\theta=0^{\circ}$ (solid blue) and $\theta=180^{\circ}$ (dashed orange). Each data point is averaged over ten measurements and the standard deviation is shown.

experiments were performed in air and single-shot ablation craters were created due to a fast translation of the sample in the direction perpendicular to the laser propagation axis (for details on LIA see the Supplemental Material [28], and Refs. [29-33] therein). The beam radius at focus $\left(1 / e^{2}\right.$ of intensity) for both 1800 and $900 \mathrm{~nm}$ fields was $\sim 17 \mu \mathrm{m}$ and their pulse duration was characterized to 65 fs from SHGfrequency-resolved optical gating measurements. The intensities for the 1800- and 900-nm fields were respectively $1.6 \times$ $10^{13}$ and $7.7 \times 10^{12} \mathrm{~W} \mathrm{~cm}^{-2}$. Shot-to-shot energy fluctuations were measured to be $2 \%$ at $1800 \mathrm{~nm}$ and $3 \%$ at $900 \mathrm{~nm}$. The craters were imaged with a Nikon Eclipse microscope $(100 \times$ objective) and their area was determined with a fitting routine whose accuracy was cross-checked by atomic force microscopy measurements [28].

\section{RESULTS AND DISCUSSION}

\section{A. Two-color LIA measurements}

We first address the following question: Can tunnel ionization in a solid be efficiently controlled as has been demonstrated in atomic and molecular gases [22-25,34,35]? To do so, we experimentally used a two-color field expressed by

$$
\mathcal{F}(t)=\mathcal{F}_{1}(t) \cos (\omega t)+\mathcal{F}_{2}(t) \cos (2 \omega t+\phi),
$$

where $\mathcal{F}_{1}$ and $\mathcal{F}_{2}$ are the envelope functions of the fundamental field and its second harmonic, of frequencies $\omega$ and $2 \omega$, respectively. By varying the relative phase $\phi$, one can shape the waveform of the total electric field under the envelope and control its polarity [Fig. 1(b)]. We therefore performed two-color LIA with the $c$ axis of the crystal parallel to the laser polarization $\left(\theta=0^{\circ}\right)$. Figure 2 shows the resulting ablated area in $\mathrm{LiNbO}_{3}$ as a function of $\phi$ for $\theta=0^{\circ}$ (blue solid curve). A clear phase dependence is observed with a periodicity that is half the fundamental period. The modulation amplitude corresponds to $35 \%$ of the mean value. From Eq. (1) the peak electric field is maximal when $\phi=0$ or $\pi$, differing solely by its polarity. The successive extrema with a $\Delta \phi=\pi$ in Fig. 2 thus demonstrate that the ablation process is sensitive to the field polarity for a laser polarization parallel 
to the crystal $c$ axis. For a process depending on the direction of the peak electric field, one would expect that rotating the sample by $180^{\circ}$ with respect to the laser polarization would revert the observed trend regardless of the mechanism. This is confirmed by the orange (dashed) curve, showing an out-ofphase dependence when repeating the measurements for $\theta=$ $180^{\circ}$ and preserving the same initial two-color phase [28]. Experimentally, such a control is achieved most efficiently in the vicinity of the fundamental field ablation threshold. For higher intensities, the contribution of avalanche ionization to LIA reduces the sensitivity to SFI, an effect that was also observed in single-color angular LIA measurements [36]. Optimal control is obtained for a 2:1 (1800 nm:900 nm) intensity ratio, while further increasing the $\mathrm{SH}$ intensity leads to a loss of modulation. Moreover, the modulation amplitude vanishes completely at $\theta=90^{\circ}$.

\section{B. Determination of the spontaneous polarization}

In our experiment, the photon energy of $0.69 \mathrm{eV}(1800$ $\mathrm{nm}$ ) is well below the $3.78 \mathrm{eV}$ experimental band gap of $\mathrm{LiNbO}_{3}[37,38]$. Together with the laser intensity employed in the experiments, this ensures that ionization is due to tunneling $(\gamma=0.57) .180^{\circ}$ apart from each other, the two studied crystal orientations possess an identical band structure [28]. Therefore, the observed $\pi$ phase shift between the curves in Fig. 2 suggests a non-negligible contribution from the spontaneous polarization of $\mathrm{LiNbO}_{3}$, which is known to be particularly high along its $c$ axis. The effect of this spontaneous polarization is comparable to an internal electric field within the material that can either add up to or subtract from the laser electric field. Consequently, the modulation amplitude in the ablated area observed in Fig. 2 is attributable to an effect from a parallel vs antiparallel spontaneous polarization relative to the laser peak electric field. Based on this assumption, we estimate the spontaneous polarization of $\mathrm{LiNbO}_{3}$ from the regression method that was used to determine the ablation threshold of the material. Figure 3 shows the ablated area of $\mathrm{LiNbO}_{3}$ with increasing fluence for the $1800-\mathrm{nm}$ field only. Experimentally, the ablation threshold fluence $F_{\text {th }}$ can be evaluated according to $A(F)=\pi / 2 r_{0}^{2} \ln \left(F / F_{\text {th }}\right)$ where $A$ is the ablated area, $r_{0}$ the effective beam radius $\left(1 / e^{2}\right)$, and $F$ the laser fluence $[39,40]$. From this fitting, we report $F_{\text {th }}=$ $0.51 \mathrm{~J} / \mathrm{cm}^{2}$ in lithium niobate at an $1800-\mathrm{nm}$ wavelength.

To retrieve the value of the effective spontaneous polarization of the material, we evaluate the peak electric field difference $\Delta \mathcal{F}$ corresponding to the fluences that would be required to reach maxima $\left(170 \mu \mathrm{m}^{2}\right)$ and minima $\left(120 \mu \mathrm{m}^{2}\right)$ in the two-color measurements, as illustrated in Fig. 3. After considering Fresnel losses of the field $\mathcal{F}$ [41], the resulting field in the material corresponds to $\mathcal{F}_{\mathrm{LiNbO}_{3}}=2 \mathcal{F} /\left(n_{e}+1\right)$, with the refractive index $n_{e}=2.13$ at $1800 \mathrm{~nm}$. A value of $\Delta \mathcal{F}_{\mathrm{LiNbO}_{3}}=$ $0.46 \mathrm{GV} / \mathrm{m}$ is obtained for which the half-value corresponds to a spontaneous polarization $\left|P_{s}\right|=0.06 \mathrm{C} / \mathrm{m}^{2}(\epsilon=2.54 \times$ $\left.10^{-10} \mathrm{~F} / \mathrm{m}\right)$ [26]. Although there is no precise technique to characterize this property, $\left|P_{S}\right|$ in $\mathrm{LiNbO}_{3}$ has been reported to range between 0.6 and $0.8 \mathrm{C} / \mathrm{m}^{2}$ from density-functional theory calculations and polarization switching measurements [42-44]. In most cases, a pure stoichiometric crystal is considered and the reported values thus correspond to an upper

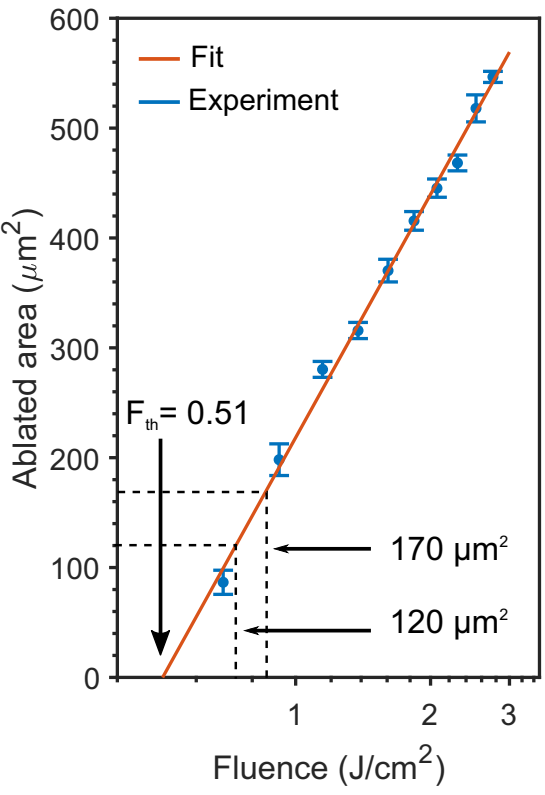

FIG. 3. Determination of the spontaneous polarization. The ablated area as a function of 1800-nm field fluence (logarithmic scale) is shown. An ablation threshold of $0.51 \mathrm{~J} / \mathrm{cm}^{2}$ is retrieved from the fit. Dashed lines show the interpolated fluences for the maximal and minimal crater area in Fig. 2 from which a spontaneous polarization of $0.06 \mathrm{C} / \mathrm{m}^{2}$ is retrieved.

limit. The reduced value retrieved in our experiments may be attributed to the initial presence of free charges within the material due to defects, intrinsically present for nonstoichiometric $\mathrm{LiNbO}_{3}$ samples. Screening by free carriers, together with surface charge density effects [45], are expected to reduce significantly the effective spontaneous polarization.

\section{Two-band model}

To get deeper insight into the underlying control mechanism, we used the experimentally retrieved $P_{s}$ to simulate the two-color phase dependence of the ionization process with a two-band model for $\mathrm{LiNbO}_{3}$. The calculations are performed by solving the semiconductor Bloch equations (for theoretical details, see the Supplemental Material [28] and references [46-50] therein). In presence of the laser field, the band gap $E_{g}$ undergoes an AC Stark shift that is described to the first order by

$$
E_{g}(\mathcal{F})=E_{g}(0)-\mu \mathcal{F}(t)_{\mathrm{LiNbO}_{3}},
$$

where $E_{g}(0)$ is the field-free band-gap energy and $\mathcal{F}(t)_{\mathrm{LiNbO}_{3}}$ is the two-color laser electric field in the material. This is in direct analogy to Stark shifts experienced by asymmetric molecules in intense laser fields [51]. The permanent dipole of $\mathrm{LiNbO}_{3}$ along its $c$ axis, $\mu$, is obtained by multiplying $P_{s}$ by the volume of the unit cell [28]. The retrieved $P_{s}=$ $\pm 0.06 \mathrm{C} / \mathrm{m}^{2}$ thus enters Eq. (2) via the permanent dipole $\mu$ and the dynamical band-gap energy is included in our twoband model. Figure 4 shows the resulting conduction band population of the material as a function of $\phi$. The two spontaneous polarizations $P_{s}= \pm 0.05 \mathrm{C} / \mathrm{m}^{2}$ and $P_{s}= \pm 0.1 \mathrm{C} / \mathrm{m}^{2}$ 


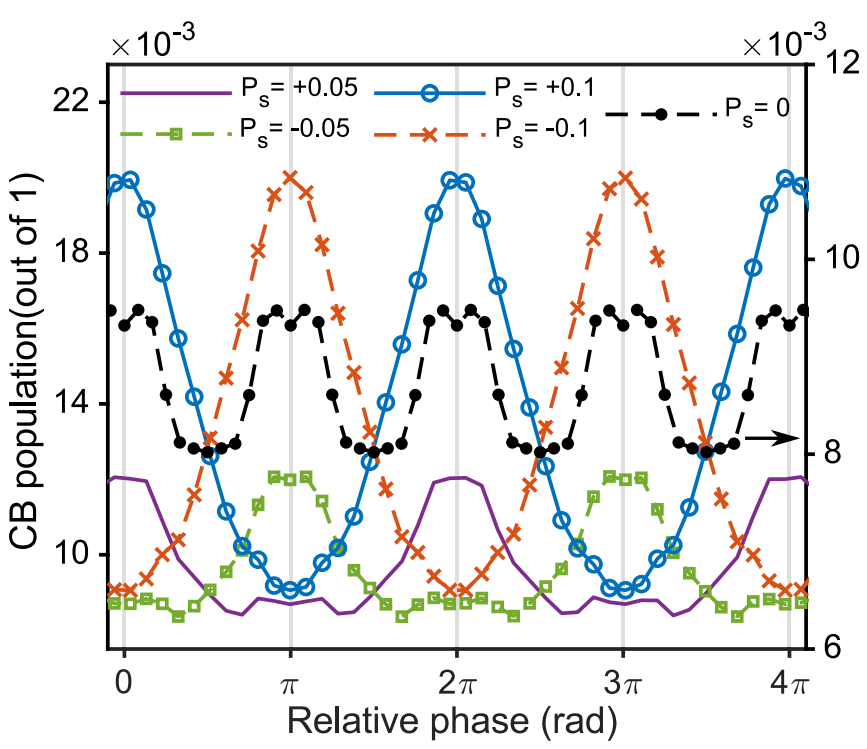

FIG. 4. Two-band model calculations. The conduction band (CB) population as a function of the relative two-color phase $\phi$ is shown for $\theta=0^{\circ}$ and $\theta=180^{\circ}$. The two orientations correspond to a sign reversal of the spontaneous polarization that is either parallel or antiparallel to the laser peak electric field. Different values are compared: $P_{s}= \pm 0.05 \mathrm{C} / \mathrm{m}^{2}$ (green squares and purple line), $P_{s}= \pm 0.1 \mathrm{C} / \mathrm{m}^{2}$ (blue circles and orange crosses), and $P_{s}=0 \mathrm{C} / \mathrm{m}^{2}$ (black dots). While a sign reversal leads to a $\pi$ phase shift of the modulation, the frequency is doubled when $P_{s}=0 \mathrm{C} / \mathrm{m}^{2}$ (black dots, right scale).

are compared. A sign reversal is equivalent to rotating the sample spatially from $\theta=0^{\circ}$ to $180^{\circ}$.

First, one can observe by comparing $P_{s}=+0.05$ and $-0.05 \mathrm{C} / \mathrm{m}^{2}$ (green squares and purple line) that reversing $P_{s}$ leads to a $\pi$-shifted modulation. Increasing to $P_{s}=$ +0.1 and $-0.1 \mathrm{C} / \mathrm{m}^{2}$ (blue circles and orange crosses), this feature is preserved and the modulation amplitude is enhanced. As described by Eq. (2), a larger spontaneous polarization $\left(\left|P_{s}\right|=0.05\right.$ vs $\left.0.1 \mathrm{C} / \mathrm{m}^{2}\right)$ amplifies the AC Stark effect on the band-gap energy [28]. Since the ionization rate depends exponentially on $E_{g}$, this leads to a significant increase of the modulation amplitude of the conduction band population. In agreement with the experimental results of Fig. 2, extrema arise every $\Delta \phi=\pi$, confirming the dependence of the ionization process on the electric field polarity. For comparison, the black curve describes ionization when the spontaneous polarization of $\mathrm{LiNbO}_{3}$ is not taken into account $\left(P_{s}=0\right)$. In this case, the modulation frequency is doubled and the process depends on $|\mathcal{F}|^{2}$, confirming the key role of the spontaneous polarization as the precursor for the symmetry breaking. The transition from $P_{s}=0$ to $0.1 \mathrm{C} / \mathrm{m}^{2}$ in the two-color phase dependence illustrates the change of periodicity induced by the material [28]. It can be noted that in the simulations (Fig. 4) an extrema is observed precisely at $\phi=0$, in agreement with Eq. (1). This is not observed in the experimental results (Fig. 2) because for this latter case the initial two-color phase $\phi$ is unknown but arbitrarily chosen as a relative zero. While our simulations provide a qualitative interpretation, additional effects should be taken into account for a quantitative modeling. For instance, indirect band-gap transitions in the tunneling regime may contribute to the conduction band population. Thermodynamic properties such as electron and ion temperatures and pressures should also be considered [11].

\section{CONCLUSION}

In summary, we demonstrated an efficient control of the tunnel ionization rate in lithium niobate from LIA measurements. By using a phase-controlled two-color laser field to ablate the ferroelectric material, we observed modulations as high as $35 \%$ in the ablated area when varying the relative phase between an 1800-nm field and its second harmonic. The results are explained by a two-band model, which clarifies the control mechanism as a consequence of the spontaneous polarization in $\mathrm{LiNbO}_{3}$. This is in close analogy to the molecular case, where, however, noncentrosymmetric molecules must be oriented in the laboratory frame in order to determine the influence of permanent dipole moment and polarizability [21]. From a regression method, we evaluated $\left|P_{s}\right|$ to be $0.06 \mathrm{C} / \mathrm{m}^{2}$. Simulations show that parallel and antiparallel spontaneous polarization relative to the laser peak electric field direction influences the AC Stark shift of the band-gap energy. This, in turn, impacts on the ionization rate of the material and leads to ablated craters of different sizes. Our experimental approach hence allows interpreting the role of the spontaneous polarization in the ionization of ferroelectric materials. Smaller band-gap materials with high $P_{s}$ should lead to enhanced control. This will, however, require midinfrared wavelength pulses with microjoules of energy to remain in the tunneling regime. The observed phase sensitivity of femtosecond laser-induced ablation can find applications in laser micromachining but most importantly it opens a route for the direct control of ionization dynamics within crystals through femtosecond pulse shaping. For example, our results offer new degrees of freedom for high-order harmonic generation from solids, a promising candidate for the design of compact extreme ultraviolet sources. Finally, the findings also suggest a potential phase meter scheme, exploiting solid samples, to identify the carrier-envelope phase of few-cycle laser pulses covering the mid- and far-IR spectral ranges gaining in accessibility in many laboratories.

\section{ACKNOWLEDGMENTS}

V.W. thanks Guilmot Ernotte for fruitful discussions about the interpretation of ionization dynamics in solids. The authors acknowledge financial support from Canada Foundation for Innovation (CFI), the Natural Sciences and Engineering Research Council of Canada (NSERC), the Fonds de recherche du Québec-Nature et technologies (FRQNT), PROMPT, and the National Natural Science Foundation of China (NSFC) (Grants No. 91850121, No. 11674363, and No. 11561121002). V.W. was supported by the Vanier Canada Graduate Scholarship (Vanier CGS) program and FRQNT. J.D. was supported by NSERC.

V.W. and T.J.S. contributed equally to this work. 
[1] P. Agostini, F. Fabre, G. Mainfray, G. Petite, and N. K. Rahman, Free-Free Transitions Following Six-Photon Ionization of Xenon Atoms, Phys. Rev. Lett. 42, 1127 (1979).

[2] S. M. Hankin, D. M. Villeneuve, P. B. Corkum, and D. M. Rayner, Intense-field laser ionization rates in atoms and molecules, Phys. Rev. A 64, 013405 (2001).

[3] L. V. Keldysh, Ionization in the field of a strong electromagnetic wave, Sov. Phys. JETP 20, 1307 (1964).

[4] P. B. Corkum, Plasma Perspective on Strong-Field Multiphoton Ionization, Phys. Rev. Lett. 71, 1994 (1993).

[5] F. Calegari, G. Sansone, S. Stagira, C. Vozzi, and M. Nisoli, Advances in attosecond science, J. Phys. B: At., Mol. Opt. Phys. 49, 062001 (2016).

[6] D. Homoelle, S. Wielandy, A. L. Gaeta, N. F. Borrelli, and C. Smith, Infrared photosensitivity in silica glasses exposed to femtosecond laser pulses, Opt. Lett. 24, 1311 (1999).

[7] S. Ghimire, A. D. Dichiara, E. Sistrunk, P. Agostini, L. F. Dimauro, and D. A. Reis, Observation of high-order harmonic generation in a bulk crystal, Nat. Phys. 7, 138 (2011).

[8] G. Vampa, T. J. Hammond, N. Thiré, B. E. Schmidt, F. Légaré, C. R. McDonald, T. Brabec, and P. B. Corkum, Linking high harmonics from gases and solids, Nature (London) 522, 462 (2015).

[9] N. Bloembergen, Laser induced electric breakdown in solids, IEEE J. Quantum Electron. 10, 375 (1974).

[10] C. B. Schaffer, A. Brodeur, and E. Mazur, Laser-induced breakdown and damage in bulk transparent materials induced by tightly focused femtosecond laser pulses, Meas. Sci. Technol. 12, 1784 (2001).

[11] B. Chimier, O. Uteza, N. Sanner, M. Sentis, T. Itina, P. Lassonde, F. Legare, F. Vidal, and J. C. Kieffer, Damage and ablation thresholds of fused-silica in femtosecond regime, Phys. Rev. B 84, 094104 (2011).

[12] M. Gertsvolf, H. Jean-Ruel, P. P. Rajeev, D. D. Klug, D. M. Rayner, and P. B. Corkum, Orientation-Dependent Multiphoton Ionization in Wide Band Gap Crystals, Phys. Rev. Lett. 101, 243001 (2008).

[13] S. Ghimire, A. D. DiChiara, E. Sistrunk, U. B. Szafruga, P. Agostini, L. F. DiMauro, and D. A. Reis, Redshift in the Optical Absorption of ZnO Single Crystals in the Presence of an Intense Midinfrared Laser Field, Phys. Rev. Lett. 107, 167407 (2011).

[14] A. V. Mitrofanov, A. J. Verhoef, E. E. Serebryannikov, J. Lumeau, L. Glebov, A. M. Zheltikov, and A. Baltuška, Optical Detection of Attosecond Ionization Induced by a Few-Cycle Laser Field in a Transparent Dielectric Material, Phys. Rev. Lett. 106, 147401 (2011).

[15] S. M. Golin, S. E. Kirkwood, D. D. Klug, D. M. Villeneuve, D. M. Rayner, C. A. Herrero, and P. B. Corkum, Strong field processes inside gallium arsenide, J. Phys. B 47, 204025 (2014).

[16] A. Schiffrin, T. Paasch-Colberg, N. Karpowicz, V. Apalkov, D. Gerster, S. Mühlbrandt, M. Korbman, J. Reichert, M. Schultze, S. Holzner, J. V. Barth, R. Kienberger, R. Ernstorfer, V. S. Yakovlev, M. I. Stockman, and F. Krausz, Optical-field-induced current in dielectrics, Nature (London) 493, 70 (2013).

[17] H. Liang, P. Krogen, Z. Wang, H. Park, T. Kroh, K. Zawilski, P. Schunemann, J. Moses, L. F. Dimauro, F. X. Kärtner, and K.-h. Hong, High-energy mid-infrared sub-cycle pulse synthesis from a parametric amplifier, Nat. Commun. 8, 141 (2017).
[18] A. Guarino, G. Poberaj, D. Rezzonico, R. Degl'Innocenti, and P. Günter, Electro-optically tunable microring resonators in lithium niobate, Nat. Photonics 1, 407 (2007).

[19] B. J. Eggleton, B. Luther-Davies, and K. Richardson, Chalcogenide photonics, Nat. Photoncs 5, 141 (2011).

[20] H. Akagi, T. Otobe, A. Staudte, A. Shiner, F. Turner, R. Dorner, D. M. Villeneuve, and P. B. Corkum, Laser tunnel ionization from multiple orbitals in $\mathrm{HCl}$, Science 325, 1364 (2009).

[21] L. Holmegaard, J. L. Hansen, L. Kalhøj, S. Louise Kragh, H. Stapelfeldt, F. Filsinger, J. Küpper, G. Meijer, D. Dimitrovski, M. Abu-Samha, C. P. Martiny, and L. Bojer Madsen, Photoelectron angular distributions from strong-field ionization of oriented molecules, Nat. Phys. 6, 428 (2010).

[22] H. Li, D. Ray, S. De, I. Znakovskaya, W. Cao, G. Laurent, Z. Wang, M. F. Kling, A. T. Le, and C. L. Cocke, Orientation dependence of the ionization of $\mathrm{CO}$ and NO in an intense femtosecond two-color laser field, Phys. Rev. A 84, 043429 (2011).

[23] H. Ohmura, N. Saito, and T. Morishita, Molecular tunneling ionization of the carbonyl sulfide molecule by double-frequency phase-controlled laser fields, Phys. Rev. A 89, 013405 (2014).

[24] Q. Liao, P. Lu, P. Lan, W. Cao, and Y. Li, Phase dependence of high-order above-threshold ionization in asymmetric molecules, Phys. Rev. A 77, 013408 (2008).

[25] G. L. Kamta and A. D. Bandrauk, Phase Dependence of Enhanced Ionization in Asymmetric Molecules, Phys. Rev. Lett. 94, 203003 (2005).

[26] R. S. Weis and T. K. Gaylord, Lithium niobate: Summary of physical properties and crystal structure, Appl. Phys. A 37, 191 (1985).

[27] T. Endo, H. Fujise, Y. Kawachi, A. Ishihara, A. Matsuda, M. Fushitani, H. Kono, and A. Hishikawa, Selective bond breaking of $\mathrm{CO}_{2}$ in phase-locked two-color intense laser fields: Laser field intensity dependence, Phys. Chem. Chem. Phys. 19, 3550 (2017).

[28] See Supplemental Material at http://link.aps.org/supplemental/ 10.1103/PhysRevB.101.184103 for details on the theoretical model and data analysis as well as complementary results and simulations.

[29] X. Liu, D. Du, and G. Mourou, Laser ablation and micromachining with ultrashort laser pulses, IEEE J. Quantum Electron. 33, 1706 (1997).

[30] B. C. Stuart, M. D. Feit, S. Herman, A. M. Rubenchik, B. W. Shore, and M. D. Perry, Optical ablation by high-power shortpulse lasers, J. Opt. Soc. Am. B 13, 459 (1996).

[31] B. C. Stuart, M. D. Feit, S. Herman, A. M. Rubenchik, B. W. Shore, and M. D. Perry, Nanosecond-to-femtosecond laserinduced breakdown in dielectrics, Phys. Rev. B 53, 1749 (1996).

[32] F. Vidal, T. W. Johnston, S. Laville, O. Barthélemy, M. Chaker, B. Le Drogoff, J. Margot, and M. Sabsabi, Critical-Point Phase Separation in Laser Ablation of Conductors, Phys. Rev. Lett. 86, 2573 (2001).

[33] F. Seitz, On the theory of electron multiplication, Phys. Rev. 76, 1376 (1949).

[34] S. Watanabe, K. Kondo, Y. Nabekawa, A. Sagisaka, and Y. Kobayashi, Two-Color Phase Control in Tunneling Ionization and Harmonic Generation by a Strong Laser Field and its Third Harmonic, Phys. Rev. Lett. 73, 2692 (1994). 
[35] D. W. Schumacher, F. Weihe, H. G. Muller, and P. H. Bucksbaum, Phase Dependence of Intense Field Ionization: A Study Using Two Colors, Phys. Rev. Lett. 73, 1344 (1994).

[36] X. Li, W. Rong, L. Jiang, K. Zhang, C. Li, Q. Cao, G. Zhang, and Y. Lu, Generation and elimination of polarizationdependent ablation of cubic crystals by femtosecond laser radiation, Opt. Express 22, 30170 (2014).

[37] C. Thierfelder, S. Sanna, A. Schindlmayr, and W. G. Schmidt, Do we know the band gap of lithium niobate? Phys. Status Solidi C 7, 362 (2010).

[38] A. Riefer, M. Friedrich, S. Sanna, U. Gerstmann, A. Schindlmayr, and W. G. Schmidt, $\mathrm{LiNbO}_{3}$ electronic structure: Many-body interactions, spin-orbit coupling, and thermal effects, Phys. Rev. B 93, 075205 (2016).

[39] J. M. Liu, Simple technique for measurements of pulsed Gaussian-beam spot sizes, Opt. Lett. 7, 196 (1982).

[40] O. Uteza, B. Bussière, F. Canova, J. P. Chambaret, P. Delaporte, T. Itina, and M. Sentis, Laser-induced damage threshold of sapphire in nanosecond, picosecond and femtosecond regimes, Appl. Surf. Sci. 254, 799 (2007).

[41] G. Vampa, C. R. McDonald, G. Orlando, P. B. Corkum, and T. Brabec, Semiclassical analysis of high harmonic generation in bulk crystals, Phys. Rev. B 91, 064302 (2015).

[42] S. R. Phillpot and V. Gopalan, Coupled displacive and orderdisorder dynamics in $\mathrm{LiNbO}_{3}$ by molecular-dynamics simulation, Appl. Phys. Lett. 84, 1916 (2004).
[43] V. Gopalan, T. E. Mitchell, Y. Furukawa, and K. Kitamura, The role of nonstoichiometry in $180^{\circ}$ domain switching of $\mathrm{LiNbO}_{3}$ crystals, Appl. Phys. Lett. 72, 1981 (1998).

[44] D. Lee, H. Xu, V. Dierolf, V. Gopalan, and S. R. Phillpot, Structure and energetics of ferroelectric domain walls in $\mathrm{LiNbO}_{3}$ from atomic-level simulations, Phys. Rev. B 82, 014104 (2010).

[45] F. Johann and E. Soergel, Quantitative measurement of the surface charge density, Appl. Phys. Lett. 95, 232906 (2009).

[46] A. Dhar and A. Mansingh, Optical properties of lithium niobate single crystals, J. Appl. Phys. 68, 5804 (1990).

[47] G. Kresse and J. Hafner, Ab initio molecular dynamics for liquid metals, Phys. Rev. B 47, 558 (1993).

[48] P. E. Blöchl, Projector augmented-wave method, Phys. Rev. B 50, 17953 (1994).

[49] C. R. McDonald, G. Vampa, P. B. Corkum, and T. Brabec, Intense-Laser Solid State Physics: Unraveling the Difference between Semiconductors and Dielectrics, Phys. Rev. Lett. 118, 173601 (2017).

[50] W. Kohn and L. J. Sham, Self-consistent equations including exchange and correlation effects, Phys. Rev. 140, A1133 (1965).

[51] A. Etches and L. B. Madsen, Extending the strong-field approximation of high-order harmonic generation to polar molecules: Gating mechanisms and extension of the harmonic cutoff, J. Phys. B: At., Mol. Opt. Phys. 43, 155602 (2010). 